\title{
Cross-Cultural Differences in Children's Beliefs About the Objectivity of Social Categories
}

\author{
Gil Diesendruck and Rebecca Goldfein-Elbaz \\ Bar-Ilan University
}

Susan Gelman

University of Michigan

\author{
Marjorie Rhodes \\ New York University \\ Noam Neumark \\ New York University
}

\begin{abstract}
The present study compared 5- and 10-year-old North American and Israeli children's beliefs about the objectivity of different categories $(n=109)$. Children saw picture triads composed of two exemplars of the same category (e.g., two women) and an exemplar of a contrasting category (e.g., a man). Children were asked whether it would be acceptable or wrong for people in a different country to consider contrasting exemplars to be the same kind. It was found that children from both countries viewed gender as objectively correct and occupation as flexible. The findings regarding race and ethnicity differed in the two countries, revealing how an essentialist bias interacts with cultural input in directing children's conceptualization of social groups.
\end{abstract}

A growing number of studies reveal that not only do children manifest intergroup biases from a young age (Bigler \& Liben, 2007; Dunham, Baron, \& Carey 2011; Rutland, Killen, \& Abrams 2010), but moreover their group concepts are couched in essentialist terms (Gelman, 2003). Specifically, children seem to conceive of certain social categories, such as race, ethnicity, or gender, as being permanent, biologically determined, inductively powerful, and as capturing objectively "natural" kinds. Research on adults has shown both positive correlational and experimental connections between an essentialist conceptualization of certain social categories (but not all, see Haslam, Rothschild, \& Ernst 2002), and negative attitudes toward that category (see Prentice \& Miller, 2007, for a review, and Keller, 2005; Morton, Postmes, Haslam, \& Hornsey 2009; Williams \& Eberhardt, 2008, for supporting evidence). Whereas no direct links between essentialism and attitudes has yet been shown among children, the two phenomena show similar developmental trends. For instance, North American

This research was funded by Grant 672/09 awarded by the Israel Science Foundation to Gil Diesendruck, New York University Challenge Funds to Marjorie Rhodes, and NICHD Grant HD-36043 to Susan Gelman. The study was part of Rebecca Goldfein-Elbaz's master's thesis. We want to thank the teachers, parents, and children for their participation.

Correspondence concerning this article should be addressed to Gil Diesendruck, Department of Psychology, Bar-Ilan University, Ramat-Gan 52900, Israel. Electronic mail may be sent to dieseng@mail.biu.ac.il. kindergarteners reveal implicit attitudes regarding race-conceptualized strictly in terms of Blacks and Whites (Baron \& Banaji, 2006), and they view race as an inheritable characteristic (Hirschfeld, 1996). Analogously, Jewish Israeli kindergarteners hold negative stereotypes regarding Arabs (Bar-Tal \& Teichman, 2005), and they view ethnicityconceptualized as Jews and Arabs-as an inductively powerful category, more so than gender, social status, religiosity, or various personality traits (Diesendruck \& haLevi, 2006).

As hinted earlier, whereas essentialist thinking about social categories seems to be present in various cultures, the particular type of category essentialized varies. For instance, in addition to the examples earlier regarding race in the United States and ethnicity in Israel, North American kindergarteners treat gender as biologically, rather than environmentally, determined (Taylor, Rhodes, \& Gelman 2009), Chilean children view social class as inductively powerful (del Rio \& Strasser, 2011), and Vezo children in Madagascar conceive of ethnicity as inheritable (Astuti, Solomon, \& Carey 2004). These findings of cross-cultural variation could be taken to present an interesting challenge to explaining the development of social essentialism.

(C) 2013 The Authors

Child Development (c) 2013 Society for Research in Child Development, Inc. All rights reserved. 0009-3920/2013/8406-0009

DOI: $10.1111 /$ cdev. 12108 
On the one hand, if different cultures vary in terms of which social categories invoke essentialist beliefs, then we could conclude that cultural input plays an important role in the developmental process. On the other hand, the sheer universality of social essentialism could be taken to indicate that cultural input in fact plays a minimal role. Crucially, however, before we can adjudicate between these possibilities, we need to ascertain that the cross-cultural variability is not due to simple differences in the very categories assessed. To this end, we need to evaluate essentialist beliefs of children from different cultures regarding a variety of similar categories. That is the main goal of this study.

To date, relevant studies have compared how children from different subpopulations within a country conceptualize the same type of social category. For instance, it has been found that in Israel, already by kindergarten, religious Jewish children attribute higher inductive potential to ethnicity than do Muslim Arab or secular Jewish children (Birnbaum, Deeb, Segall, Ben-Eliyahu, \& Diesendruck 2010), North American teenagers from rural homogenous communities view race and gender as a natural category to a greater extent than do children from urban diverse cities (Rhodes \& Gelman, 2009), and African American kindergarteners view race as more stable than do European American children (Kinzler \& Dautel, 2012). This study takes a step further and assesses how children from different countries view the same social categories and how this process might change as children become immersed in their cultures.

The comparison being tested here will provide clearer conclusions regarding the theoretical positions on the development of essentialist beliefs, alluded earlier. Some scholars have suggested that cultural values and input are crucial for the very elaboration of essentialist thinking (Fodor, 1998). A milder role of culture is proposed by others who argue that although children might have an intuitive tendency to essentialize biological kinds, the generalization of such a tendency toward social kinds is culturally mediated (Atran, 1995; GilWhite, 2001). An even more modest - though still important-role of culture is attributed by those who claim that although children may be innately predisposed to divide the social world into essentially distinct kinds, the specific kinds that end up being essentialized are defined by cultural input (Hirschfeld, 1996).

Especially in light of the latter two accounts, a question arises as to whether there are unique or necessary features that make particular social categories optimal candidates for essentialization (see Kinzler, Shutts, \& Correll 2010, for a parallel question). For instance, Atran (1995) suggests that morphological distinctiveness may reinforce the essentialization of racial categories in the United States, and Gil-White (2001) argues that the practice of endogamous weddings and the intergenerational transmission of group membership reinforce the essentialization of ethnicity in Mongolia. Others have noted more generally that category types that either mark coalitional alliances (e.g., those involved in interethnic conflicts) or have evolutionary significance (e.g., gender) are primary targets for essentialization (Cosmides, Tooby, \& Kurzban 2003). Finally, it has also been suggested that children might be particularly prone to form conceptually rich categories of social groups defined by deontic or role-based properties such as occupational categories (e.g., doctors), which are defined by expectations about what category members should do (Kalish \& Lawson, 2008). To adequately address these possibilities, and more generally address the universality of the development of essentialism, it is imperative to: (a) compare children's concepts of different types of categories and (b) conduct such a comparison among children from different cultures. This study does that.

The study included children from two countries: the United States and Israel. One of the main reasons for conducting this particular comparison is that although there are certain similarities between these countries in general cultural characteristics (e.g., both are Western countries with diverse populations), the two differ in the relative prominence of certain social categories. In particular, as reviewed earlier, research in the United States has shown that the category of race becomes essentialized with development (Hirschfeld, 1996; Rhodes \& Gelman, 2009), whereas research in Israel reveals that ethnicity (i.e., categorization of Jews and Arabs) possesses that status already by kindergarten age (Birnbaum et al., 2010; Diesendruck \& haLevi, 2006). Importantly, although both these categories are present in the two countries, they likely have different meanings associated with them. For instance, in Israel, race is most salient with respect to Ethiopian Jews who have immigrated to Israel in the past two decades. In other words, race is subsumed under a culturally very salient superordinate category-Jews. In contrast, in the United States, the difference between Jews and Arabs may be seen not as an ethnic divide but rather as a difference in religion. 
A further reason to focus on these two categories is that not only do they differ in their cultural salience but they also are marked by theoretically relevant different characteristics. Namely, whereas racial categories roughly correspond to differences in intrinsic physical features such as skin color (but see Hirschfeld, 1996, for limitations of such cues), ethnic categories are often not visually apparent in intrinsic features of a person and instead are distinguished based on cultural practices, alliances, and endogamy (Gil-White, 2001). In other words, these two kinds of categories constitute prime test cases for addressing some of the positions reviewed earlier regarding the nature of candidate social categories for essentialization. Namely, if essentialism is invoked by the presence of morphologically distinct markers, then we might expect children from both countries to be equally likely to essentialize racial categories. In turn, if essentialism is driven by a need to track cultural-specific alliances maintained via endogamy and cultural practices, then children's essentialist beliefs will be directed to the social categories especially salient in their cultural environments: race in the United States, ethnicity in Israel. To more exhaustively cover the various categorical features noted above as potentially prompting essentialist thinking, we also included categories of gender and occupation. In particular, gender marks a category with strong evolutionary significance, whereas occupation is primarily defined by deontic properties.

The task used to assess children's conceptualization of categories was adapted from one previously employed by Rhodes and Gelman (2009) in their study of North American children's concepts of gender and race. The task assesses to what extent children view categories as arbitrary and culturally variable as opposed to objective and universal. In this task, children were shown triads of pictures depicting two exemplars of a given category (e.g., two women), and an exemplar from a contrasting category (e.g., a man). After establishing that children recognized the two exemplars as being indeed members of the same category, children were told about a hypothetical country where people categorize these same items differently, such that they consider the two contrasting exemplars (e.g., a man and a woman) as members of the same category. The critical question asked children whether such alternative categorization was acceptable, or was wrong and thus needed to be corrected. The rationale is that if children essentialize a given category type, then they should believe that there is only one objective and correct way to categorize. In other words, they should consider an alternative categorization of such a category type as "wrong."

We studied children from two age groups: 5- and 10-year-olds. These ages were chosen because past research on a number of the social categories assessed here have shown important developmental changes between these ages in the degree to which children essentialize these categories (e.g., see Birnbaum et al., 2010, on ethnicity; Rhodes \& Gelman, 2009, on race; Taylor et al., 2009, on gender). Our main interest was in uncovering similarities and differences in North American and Israeli children's concepts of the various social categories, and how these concepts might change with development. Of special interest were children's concepts of race and ethnicity. The hypotheses were that if racial categories become essentialized in the United States and ethnic categories in Israel because of their particular cultural salience, then we should expect children in the United States to essentialize race more than children in Israel, and children in Israel to essentialize ethnicity more than children in the United States. Alternatively, if the essentialization of race and ethnicity derive from their intrinsic characteristics as described earlier, then we should find similar patterns of essentialism regarding these two categories in both countries.

\section{Method}

\section{Participants}

A total of 109 children participated in this study: 58 in Israel and 51 in the United States. The Israeli sample consisted of secular Jewish Hebrew-speaking children from cities in central Israel. There were 29 kindergarteners $\left(M_{\text {age }}=5.9\right.$ years, range $=5.4$ 6.7 years; 13 boys and 16 girls) and 29 fifth graders $\left(M_{\text {age }}=10.6\right.$ years, range $=9.8-11.6$ years; 16 boys and 13 girls). Israeli children were recruited and tested in their kindergartens and schools. Representative of the population of the cities from which the samples were drawn, all children attending the kindergartens and schools were Jewish, from various cultural backgrounds (e.g., European, North African, and Middle Eastern descent). The U.S. sample consisted of English-speaking children from New York City, recruited and tested in either the Children's Museum of Manhattan or a public elementary school. Children in the sample came from across New York City and the sample was approximately 60\% European American, 22\% Hispanic, 8\% African American, and 10\% Asian American. There were 25 kindergarteners $\quad\left(M_{\text {age }}=5.4\right.$ years, range $=4.9-6.0$ 
years; 13 male, 12 female) and 26 fifth graders $\left(M_{\text {age }}=10.5\right.$ years, range $=9.0-11.6$ years; 13 male, 13 female). Signed parental permission was obtained for all participants in both countries.

\section{Design}

Due to difference in the settings where children from the two countries were tested, there was a slight variation in the structure of the testing sessions. In Israel, children were tested in a quiet room near their classroom, and completed four items for each of six types of categories (animals, artifacts, gender, race, ethnicity, and occupation), thus totaling 24 items. In the United States, children were tested in a separate room within a children's museum. The room was completely quiet, and was fitted for the sole purpose of this experiment. Moreover, we coded offline videos of the children and would have excluded children who either were too distracted or were interrupted by noises coming from outside the room; however, no child needed to be excluded for these reasons. In any case, to minimize the time we kept children away from the many museum exhibits, each U.S. child saw only half of the six category types, thus totaling 12 items per child. For this purpose, category types were split into two sets. Each set included one of the nonsocial types (Set A: animals, Set B: artifacts), one of the physical-based social category types (Set A: gender, Set B: race), and one of the symbol- or clothingbased social category types (Set A: occupation, Set B: ethnicity). Both 5- and 10-year-olds were randomly assigned to one of the two sets (A or B), and category types within each set were presented in counterbalanced orders across children. Children in both countries were asked the same three questions about each item in a fixed order: categorization, possibility, and objectivity (described in Procedure, below). The nonsocial category types (animals and artifacts) were included because previous work had found that 5-year-olds construe animal categories as more objective than artifact categories (Rhodes \& Gelman, 2009). Thus, we hoped that by including these two types, we would reinforce in children the possibility of rating the social categories across a wide range of objectivity.

\section{Materials}

The materials were colored photographs taken from online open sources, presented via PowerPoint in laptop computers (see Figure 1 for examples). The same pictures were used in both locations.
Exemplars for the category race were African American adults. The category ethnicity was represented by adults wearing traditional Muslim Arab and Orthodox Jewish attire (e.g., head shawls vs. yarmulke and prayer shawl, respectively). The category occupation was represented via the uniforms and accessories typically worn by doctors and police officers.

Each category type was represented by four triads. In each triad, two of the pictures were the test exemplars, and they represented instances of two contrasting categories (e.g., a man and a woman for the category type gender). The third picture was the target picture, which matched only one of the two test exemplars (e.g., a different woman).

\section{Race}
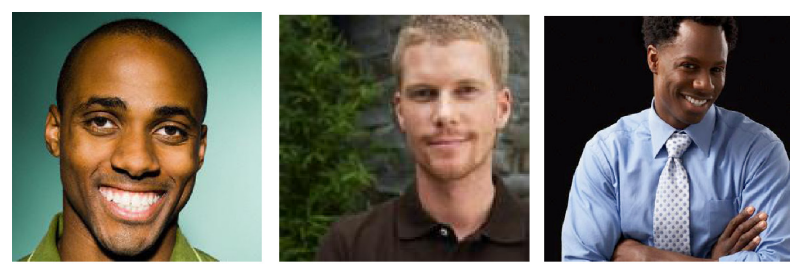

Ethnicity
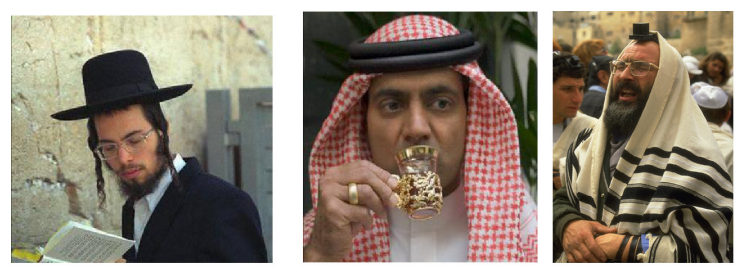

Gender
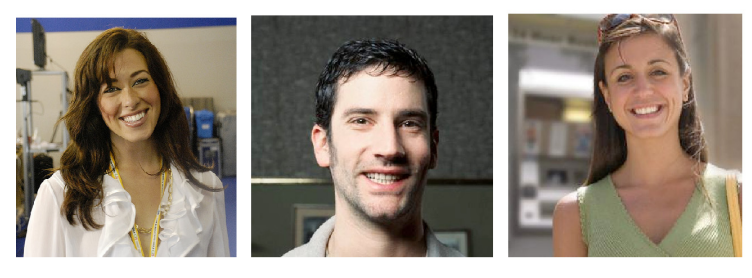

Occupation
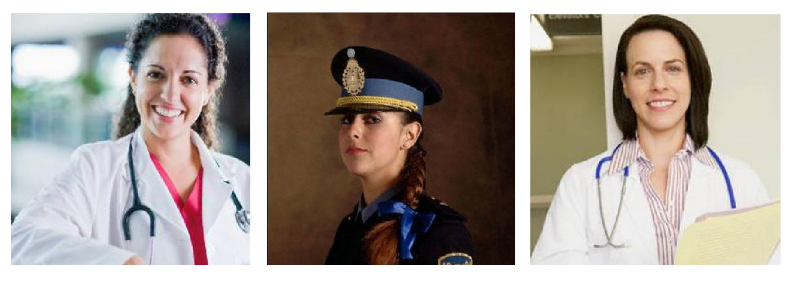

Figure 1. Examples of pictures used for the categories of people. The two left pictures were the test exemplars; the right picture was the target exemplar. In the presentation to the children, the target was placed below the test exemplars. 
The target picture always appeared below the test exemplars, and equidistant from both. The exemplars on each triad contrasted on only the relevant category type, but across triads, there were exemplars from various types. For instance, for gender, one triad represented two Black men and a Black woman, and another represented two Arab women and an Arab man. In this way, for instance, each category was not confounded with other categories, and whereas there were many dimensions by which the three exemplars in a triad could be viewed as being similar (e.g., all three were adults), there was only one salient dimension by which they could be discriminated. Table 1 lists all categories and their respective exemplars in each triad.

In addition, two triads were used for practice trials. The first triad consisted of a brick and a feather as test exemplars, and a rock as the target exemplar. The second triad consisted of broccoli and cookies as test exemplars, and chocolate ice cream as the target exemplar.

Table 1

List of All Category Types and Category Exemplars Used in the Study

\begin{tabular}{|c|c|c|}
\hline $\begin{array}{l}\text { Category } \\
\text { type }\end{array}$ & Test exemplars & Target exemplar \\
\hline \multirow[t]{4}{*}{ Animals } & 1 Cat, dog & 1 Dog \\
\hline & 2 Sheep, pig & 2 Sheep \\
\hline & 3 Frog, turtle & 3 Frog \\
\hline & 4 Lion, Wolf & 4 Lion \\
\hline \multirow[t]{4}{*}{ Artifacts } & 1 Spoon, fork & 1 Spoon \\
\hline & 2 Table, bookshelf & 2 Table \\
\hline & 3 Saw, hammer & 3 Saw \\
\hline & 4 Dress, shirt & 4 Dress \\
\hline \multirow[t]{4}{*}{ Gender } & 1 Arab woman, Arab man & 1 Arab woman \\
\hline & 2 Black man, Black woman & 2 Black man \\
\hline & 3 White man, White woman ${ }^{a}$ & 3 White woman \\
\hline & 4 White man, White woman & 4 White woman \\
\hline \multirow[t]{4}{*}{ Race } & 1 Black woman, White woman & 1 Black woman \\
\hline & 2 Black man, White man ${ }^{a}$ & 2 Black man \\
\hline & 3 Black woman, White woman & 3 Black woman \\
\hline & 4 Black man, White man & 4 Black man \\
\hline \multirow[t]{4}{*}{ Occupation } & 1 Female doctor, policewoman ${ }^{a}$ & 1 Female doctor \\
\hline & 2 Male doctor, policeman & 2 Male doctor \\
\hline & 3 Female doctor, policewoman & 3 Female doctor \\
\hline & 4 Policeman, Male doctor & 4 Policeman \\
\hline \multirow[t]{4}{*}{ Ethnicity } & 1 Arab woman, Jewish woman & 1 Arab woman \\
\hline & 2 Arab man, Jewish man & 2 Arab man \\
\hline & 3 Arab man, Jewish man ${ }^{a}$ & 3 Jewish man \\
\hline & 4 Arab man, Jewish man & 4 Jewish man \\
\hline
\end{tabular}

a Triad depicted in Figure 1. All pictures depicted different individuals (e.g., there were four different female doctors).

\section{Procedure}

After a brief introduction, the experimenter sat the child in front of the laptop computer, and told the child that she was going to show them some pictures and ask some questions. She then proceeded with the two practice trials. The goals of the practice trials were to: (a) familiarize children with the questions and (b) illustrate to the child the range of possible answers to the questions. For these purposes, the first trial asked children to consider the veracity and universality of a physical law, and the second trial asked about personal taste preferences.

Practice trials. For the first practice trial, the experimenter pointed to the brick and the feather and asked the child: "Which of these two things sinks in the water, like this thing [pointing to the rock]?" After the child responded, the experimenter asked the child: "Do you think there might be a place in the world where people say the opposite? That is, that instead of saying this thing [the brick] sinks in the water, they say that this thing [the feather] sinks in the water?" Finally, the experimenter asked the last question: "I know a place in the world where people do say the opposite from us. That is, they say that this thing [the feather] sinks in the water, and not that this thing [the brick] does. Do you think it is wrong how they do it, and that we should correct them and teach them to do it like us? Or is it OK the way they do it, just different from us?"

The second practice trial was identical, except the experimenter asked children questions about what is tasty like chocolate ice cream: broccoli or cookies, and then asked about the universality of this opinion. On both practice trials, if children answered incorrectly, they were corrected by the experimenter so that the full range of possible answers was made available to them. Specifically, on the first practice trial, the experimenter noted that it is not possible for a brick not to sink, and on the second trial, the experimenter noted that it is quite possible that people have different tastes and think that broccoli is as good as chocolate ice cream. In this manner, the practice trials exposed children to the possibility that in some cases, it is appropriate to answer the experimental questions by saying that one needs to correct what people somewhere else do because it is wrong, whereas in other cases is it appropriate to say that it is OK how others do it even if it is different from what the child thinks.

Experimental trials. Upon completion of the practice trials, the experimenter told children that they 
were now going to see pictures of things, animals, and people. The structure of the experimental trials was very similar to that of the practice trials. The experimenter presented children each triad of a given category type and asked them three questions about it.

The first question was the categorization question. The experimenter pointed to the two test exemplars (e.g., a man and a woman in a gender triad), and asked children: "Which of these two is of the same kind as this one [the test exemplar, another woman]?" Children received a score of 1 if they picked the test exemplar from the same category (e.g., the woman), and 0 if they picked the other test exemplar (e.g., the man).

Next the experimenter asked children the possibility question. This question was used exclusively as a probe to the theoretically central objectivity question. The possibility question was exactly as the one in the practice trials, that is, "Do you think there might be a place in the world where people say the opposite? That is, that instead of saying that these two people [the two women] are of the same kind, they say that these two people [the test man and the target woman] are of the same kind?" Given that this was intended as a lead-in question and did not add conceptually to the objectivity question, we did not analyze children's responses to it.

Finally, the experimenter asked the objectivity question: "I know a place in the world where people say the opposite from us. That is, instead of saying that these two people [the two women] are of the same kind (in Hebrew: "oto sug"), they say that these two people [the test man and the target woman] are of the same kind. Do you think it is wrong how they do it, and that we should correct them and teach them to do it like us? Or is it OK the way they do it, just different from us?" Children's responses were scored as 1 if they answered, "It is wrong," and 0 if they said, "It is OK." A high score on this question meant that children had an essentialist view regarding the categorization of the specific category type, and believed their categorization to be universal, normative, and an objective reflection of reality. For children who answered the categorization question in an unexpected manner (e.g., said that a man and a woman are of the same kind), the experimenter phrased the possibility and objectivity questions in a manner consistent with the child's response (e.g., asked children about a place where they consider the two women as being of the same kind). However, as described next in the Results section, we did not include data from these trials in the analyses.
Children saw all four triads for each category type in sequence, and then moved on to another type. Order of presentation of the category types, and of the triads within each type, was randomized, and counterbalanced across children. Placement of each test exemplar on the participant's right or left was counterbalanced for each triad across participants. Preliminary analyses revealed no significant effects of the categories' presentation order, either in Israel ( $p s>4)$ or in the United States $(p s>.3)$.

\section{Results}

Given the differences in design across the two countries, our first analyses were conducted separately for each country. In all analyses, because children provided a series of binary responses, we analyzed the data with binomial regression models. These yield Wald $\chi^{2}$ values as indicators of main effects and interactions. We present all data as probabilities of a particular response (probability of categorizing based on the expected criteria; probability of rejecting the alternate category).

\section{Categorization Question}

First, we examined children's responses to the categorization question, which asked children to identify two items that were of the "same kind." As shown in Table 2, children of both age groups and from both countries categorized based on the expected criteria more often than expected by chance $(50 \%)$ for each type of category ( $p$ s $<.01$, on binomial tests).

Table 2

Probabilities (and Confidence Intervals) of Categorizing Based on the Expected Criteria (Categorization Question) for Each Category Type

\begin{tabular}{llllll}
\hline & \multicolumn{2}{c}{ United States } & & \multicolumn{2}{c}{ Israel } \\
\cline { 2 - 3 } \cline { 5 - 6 } & 5-year-olds & 10-year-olds & & 5-year-olds & 10-year-olds \\
\hline Animals & $.92(.75, .98)$ & $.91(.66, .98)$ & & $.88(.81, .93)$ & $.98(.94,1)$ \\
Artifacts & $.84(.69, .93)$ & $.98(.86,1)$ & & $.95(.87, .98)$ & $.91(.85, .95)$ \\
Gender & $.80(.63, .90)$ & $.88(.65, .96)$ & & $.93(.87, .97)$ & $.94(.84, .98)$ \\
Race & $.70(.53, .83)$ & $.95(.75, .99)$ & & $.83(.74, .89)$ & $.93(.85, .97)$ \\
Occupation & $.92(.75, .98)$ & $.93(.63, .99)$ & & $.93(.87, .97)$ & $.98(.89,1)$ \\
Ethnicity & $.86(.77, .92)$ & $.82(.74, .88)$ & & $.89(.81, .94)$ & $.99(.94,1)$ \\
\hline
\end{tabular}

Note. Probabilities are based on a score of 1 if children picked the test exemplar from the same category, and 0 if they picked the exemplar from the other category. 
United States. There were no significant main effects or interaction of age and category type among children in the United States.

Israel. There were a number of significant effects among children in Israel. First, there was a significant effect of age, Wald $\chi^{2}(1)=8.19$, $p=.004$, such that older children $(M=.97$, $\mathrm{CI}=.94, .98)$ categorized more accurately than younger children $(M=.91, \mathrm{CI}=.87, .94)$. Second, there was a significant effect of category type, Wald $\chi^{2}(5)=14.57, \quad p=.01$, such that children categorized less accurately for race than for animals, occupation, or ethnicity, $p s<.05$. Finally, there was also an interaction between age and category type, Wald $\chi^{2}(5)=15.69, p=.008$. At age 5, children were less accurate for race than for gender, occupation, or artifacts, $p s<.05$. At age 10, children were less accurate for artifacts than for animals, occupation, or ethnicity, $p s$ $<.05$, and less accurate for race than for occupation, $p<.05$.

However, despite these variations, children from both countries were accurate on the majority of trials across all category types-responding correctly on over $70 \%$ of trials - indicating that the categories were highly familiar to children.

\section{Objectivity Question}

Our key question of interest was whether children would accept that alternate ways of categorizing could be correct. Rejecting these alternate categories as "wrong" indicates the belief that categories reflect an objectively accurate way of categorizing - an essentialist belief. To analyze this question, we calculated the proportion of trials in which children said it was wrong to categorize items differently, out of those trials in which they answered the categorization question in the expected manner (e.g., trials in which children said the two women were of the same kind, and then said that it would be wrong for other people to consider a man and a woman as being of the same kind).

United States. In the United States, there was a main effect of category type, Wald $\chi^{2}(5)=21.10$, $p=.001$, but no main or interactive effects of age. As can be seen in Figure 2, children rejected the categories for animals more than for occupation, $p=.007$, or race, $p=.05$; they rejected the categories for gender more than for occupation, $p=.008$, or race, $p=.05$; and they rejected the categories for ethnicity more than for artifacts, $p=.05$, or race, $p=.001$.

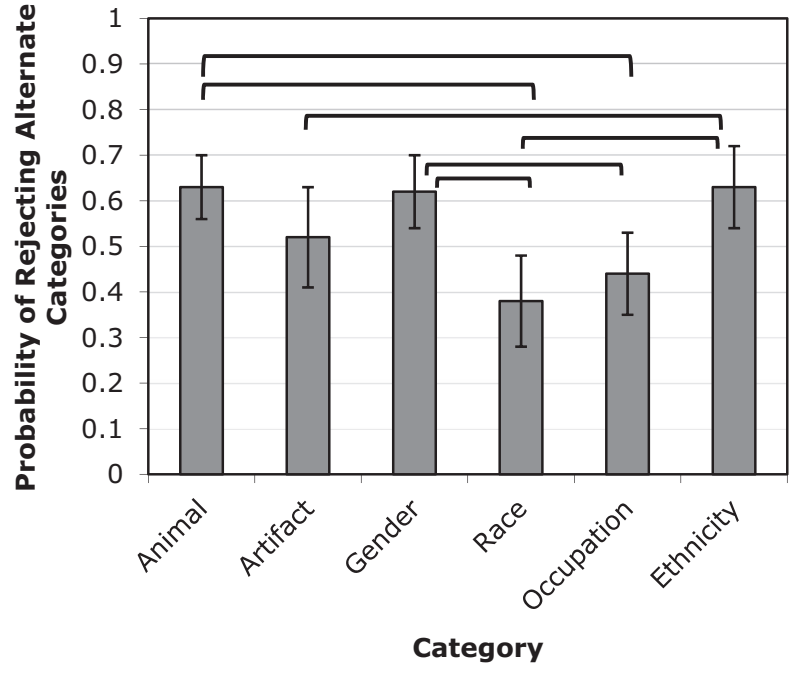

Figure 2. U.S. children's responses on the objectivity question. Error bars represent standard errors. Brackets represent significant differences between connected categories.

Israel. In Israel too there was a main effect of category type, Wald $\chi^{2}(5)=17.29, p=.004$, but no main or interactive effects of age. As can be seen in Figure 3, Israeli children rejected alternative categorization of ethnicity more than of any other category type, $p \mathrm{~s}<.05$, and they rejected the categories of gender more than race, $p=.02$.

Comparison across countries. Given the theoretical importance of the question of how essentialist beliefs about social categories vary across cultures and development, we next tested the effects of

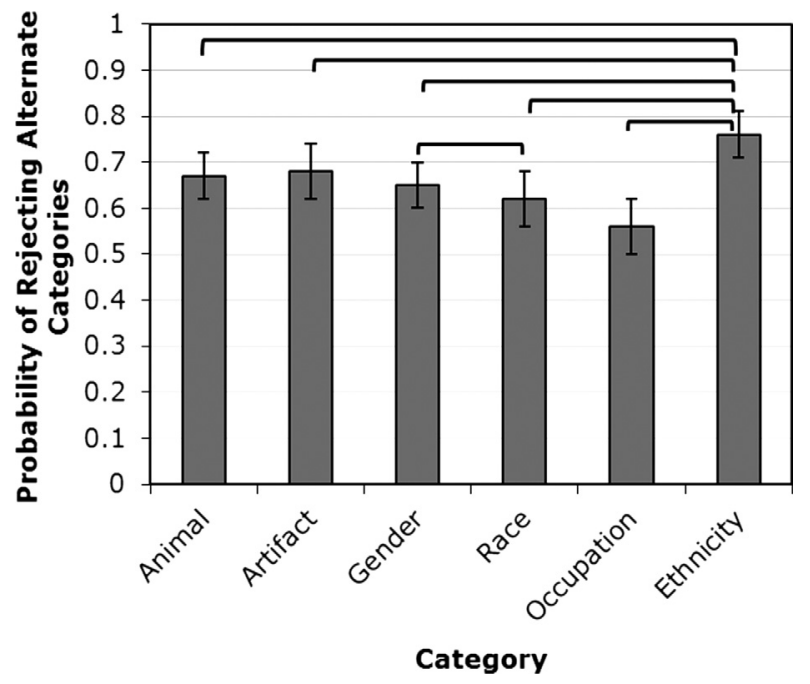

Figure 3. Israeli children's responses on the objectivity question. Error bars represent standard errors. Brackets represent significant differences between connected categories. 
Table 3

Probabilities (and Confidence Intervals) of Rejecting Alternate Categories (Objectivity Question) for the Social Categories

\begin{tabular}{llllll}
\hline & \multicolumn{2}{c}{ United States } & & \multicolumn{2}{c}{ Israel } \\
\cline { 2 - 3 } \cline { 5 - 6 } \cline { 5 - 6 } & 5-year-olds & 10-year-olds & & 5 -year-olds & 10-year-olds \\
\hline Gender & $.61(.39, .80)$ & $.63(.43, .80)$ & $.70(.55, .82)$ & $.66(.51, .78)$ \\
Race & $.25(.09, .53)$ & $.53(.27, .78)$ & $.69(.53, .81)$ & $.43(.28, .60)$ \\
Occupation & $.37(.17, .63)$ & $.52(.30, .73)$ & & $.70(.54, .82)$ & $.59(.42, .74)$ \\
Ethnicity & $.45(.22, .71)$ & $.78(.54, .92)$ & & $.76(.59, .88)$ & $.77(.60, .88)$ \\
\hline
\end{tabular}

Note. Probabilities are based on a score of 1 if children answered, "It is wrong," and 0 if they said, "It is OK."

age and country on children's objectivity beliefs about each type of social category (see means in Table 3).

For gender and occupation, there were no main or interactive effects of age or country. For race, the main effects were not significant; however, there was a significant Age $\times$ Country interaction, Wald $\chi^{2}(1)=5.62, p=.02$. As can be seen in Figure 4 , the direction of developmental change in essentialist beliefs toward race was in opposite directions in the two countries-increasing in the United States and decreasing in Israel-although the age difference reached significance only in the Israeli sample $(p=.02$ and $p=.13$, for Israel and the United States, respectively). For ethnicity, again the main effects were not significant; however, the Age $\times$ Country interaction was marginal, Wald $\chi^{2}(1)=3.03, \quad p=.08$. Essentialist beliefs about

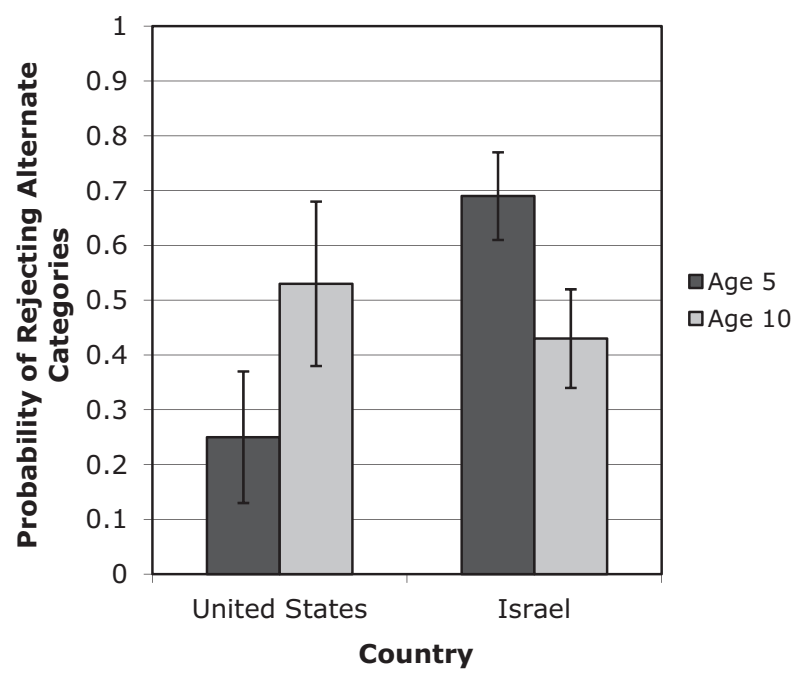

Figure 4. Comparison of U.S. and Israeli children's responses to race on the objectivity question, across ages. Error bars represent standard errors.

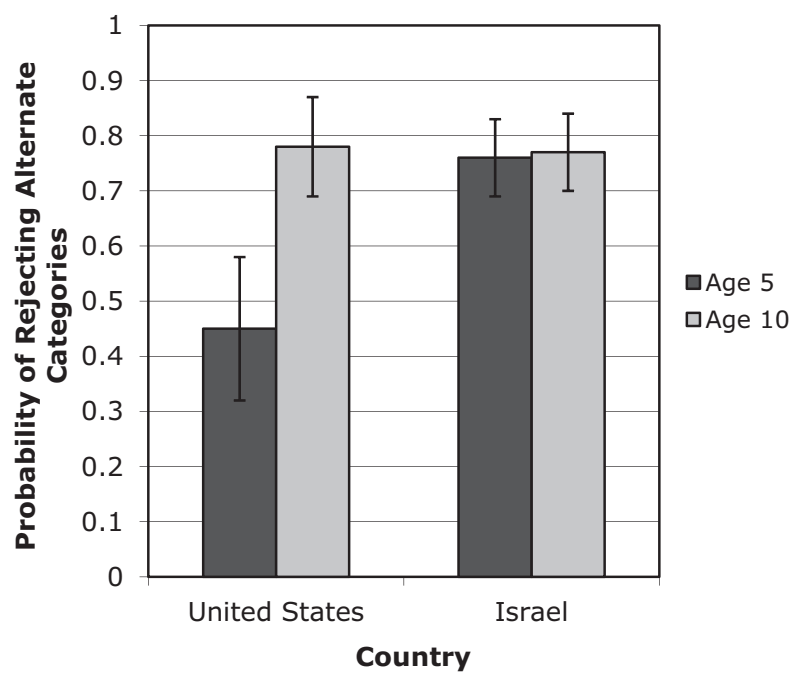

Figure 5. Comparison of U.S. and Israeli children's responses to ethnicity on the objectivity question, across ages. Error bars represent standard errors.

ethnicity increased in the United States, $p=.03$, but did not change across age in Israel, remaining high in the two age groups (see Figure 5).

\section{Discussion}

The goal of this study was to evaluate to what extent children from two different countries-United States and Israel-hold similar or different beliefs about the objectivity of various types of social categories. In particular, we were interested in assessing the role of various factors as contributors to the likelihood of children essentializing a given social category. To this end, we investigated 5- and 10-year-olds' beliefs about four types of social categories: an evolutionarily primary category type-gender; a familiar deontic category typeoccupation; a physically marked and salient category type in the United States-race; and a symbolically marked and salient category type in Israel - ethnicity. The results of the study revealed both similarities and differences in children's thinking across cultural contexts.

Regarding the similarities, we found that children in both countries tended to view gender in essentialist terms, but less so occupation. North American children across ages rejected an alternate categorization of gender more strongly than they did alternate categorizations of either race or occupation, and Israeli children rejected an alternate categorization of gender more rigorously than an alternate categorization of race. Thus, for children, 
men and women are objectively distinct kinds of people, and anyone who says otherwise should be corrected.

The relatively high essentialization of gender is consistent with previous findings that children view gender as biologically rather than environmentally determined (Taylor et al., 2009). Moreover, it supports the claim that categories with arguably high evolutionary functionality are primary candidates for essentialism (Cosmides et al., 2003). In turn, the relatively low essentialization of occupation suggests that role-based deontic categories might not be primary candidates for essentialism. An implication of this finding is that whereas children might be prone to infer deontic properties given a novel social category - as found by Kalish and Lawson (2008) - the reverse might not be true-that is, being defined by deontic properties is not sufficient in order for a familiar social category to be essentialized by children.

The similarities in the North American and Israeli children's conceptualization of gender and occupation were also important for methodological reasons. They indicate that despite differences between the two countries in the samples, testing situations, etc., the crucial objectivity question was interpreted in similar ways across countries. Consequently, differences found in children's responses to the objectivity responses could more confidently be interpreted as indicative of reliable cultural differences. Differences across countries were manifested primarily in children's beliefs about race and ethnicity. These differences were evident in both the degree of essentialism of these category types compared to other types, and in developmental changes in how they were construed.

Regarding race, two main patterns emerged. First, overall, children from both countries did not hold a highly essentialistic view of race. In particular, in both countries children were more willing to accept alternate categorizations of race than of ethnicity and gender. Second, we found opposite developmental trends in the two countries. Namely, whereas in Israel race became significantly less essentialized with age, in the United States the reverse trend was the case.

In his pioneering work on race, Hirschfeld (1996) concluded that United States and French kindergarteners already hold an essentialist conception of race. This was evident in switched-at-birth tasks, as well as tasks in which children had to decide on the heritability or stability of racial categories when contrasted with other categories. The present findings with kindergarteners would seem to contradict
Hirschfeld's conclusion. A possible reconciliation is that although young children may hold a biological view of category transmission-as assessed in Hirschfeld's tasks - they nonetheless do not regard racial categories as objectively correct-as assessed here. This explanation is consistent with the notion that young children's essentialist beliefs about human categories are not coherent (Gelman, Heyman, \& Legare 2007).

The overall developmental pattern found here with regard to race is suggestive of the importance of cultural input in the formation of children's beliefs. Namely, it seems that although children may not have an intuitive tendency to essentialize racial categories, given the "right" kind of input they learn to do so (see Cosmides et al., 2003, for a compatible conclusion). Thus, whereas North American children might be exposed to input encouraging the essentialization of race, Israeli children are not. Consistent with this interpretation are findings that North American children's tendency to treat race as a natural category (Rhodes \& Gelman, 2009) and as stable through development (Kinzler \& Dautel, 2012) increases from ages 5 to 10 years. Moreover, these developmental patterns vary across subgroups within the United States. For instance, teenagers from rural and relatively homogenous communities are more prone to embrace an essentialist conception of race than teenagers from diverse cities (Rhodes \& Gelman, 2009), and African American children give more weight to race in determining the identity of an individual than do European American children (Kinzler \& Dautel, 2012). Thus, although indirect, these latter findings intimate that the kinds of exposure children experience affect their concepts of racial categories. Taken together, the finding that racial categories were taken by children in both countries to be less objective than other social categories (e.g., gender or ethnicity), and the developmental increase in the objectivity of racial categories, intimate that these categories are not a priori prime candidates for essentialization.

The findings regarding ethnicity complement those on race. Here, we found that whereas the tendency of North American children to view ethnicity as objectively correct increased with age, Israeli children held this view already by kindergarten, with no changes through development. In fact, across ages, Israeli children rejected more strongly alternate categorizations of ethnicity than of any other category. For them, Jews and Arabs are objectively not the same kind, and those who think otherwise should be corrected. 
This high essentialist status of ethnicity among Israeli children is consistent with other studies that assessed different components of essentialist thinking. For instance, already by kindergarten, Jewish Israeli children view ethnicity as more inductively powerful (i.e., as allowing more inferences based on an individual's category membership) than other social or personality categories (Birnbaum et al., 2010; Diesendruck \& haLevi, 2006), and as stable and biologically determined (Deeb, Segall, Birnbaum, Ben-Eliyahu, \& Diesendruck 2011). Importantly, these and other studies report that although the tendency to essentialize ethnicity emerges quite early in Israeli children, it is nonetheless affected by cultural background and input. For instance, religious Jewish 5- and 6-year-olds are more likely to essentialize ethnicity than their secular age mates (Birnbaum et al., 2010; Diesendruck \& Haber, 2009), and secular Jewish second graders attending JewishArab integrated schools are less likely to essentialize ethnicity than their age mates attending Jewish-only schools (Deeb et al., 2011).

These findings suggest that the main difference found in this study between the essentialist status of ethnicity among Israeli children-high already in kindergarten - and that of race among North American children - low in kindergarten - has to do with how early the cultural input penetrates children's conceptualization of the relevant social groups. This pattern could be due to the intense interethnic conflict in Israel-and the comparably much less fervent interracial divide in New York-that makes the respective categories substantially different in their cultural salience and meaning. In particular, the sheer awareness of an antagonism between the ethnic groups might prompt Israeli children's essentialization of the groups (Cosmides et al., 2003; Rhodes \& Brickman, 2011). A further possibility is that children in Israel are exposed earlier to cultural input endorsing essentialist beliefs about ethnicity, compared to U.S. children's exposure to input about racial categories. One kind of input that has been found to endorse essentialism about social categories is certain linguistic constructions, such as generics or labels (e.g., Gelman, Taylor, \& Nguyen, 2004; Rhodes, Leslie, \& Tworek, 2012). Recent findings indeed revealed a positive correlation between Israeli parents' use of generics (e.g., "Arabs are like this") and category labels when referring to Jewish and Arab characters in a story book, and their 5year-olds' essentialist beliefs about ethnicity (Segall \& Diesendruck, 2011). A similar study evaluating North American parents' linguistic input to their children about race would provide valuable data to more directly assess the possibility raised here about cross-cultural differences in input.

An unexpected finding was that North American children regarded ethnicity as more objectively correct than race, with the significance of ethnicity increasing with age. One could have predicted that given the higher cultural relevance of race in the United States, compared to ethnicity, that the former would be more essentialized than the latter. There are a few speculative explanations of this finding. First, as noted earlier, it is possible that children in New York regarded Jews and Arabs not so much as different ethnic groups, but rather as groups engaged in different religious and cultural practices. In fact, kindergarteners treat beliefs as defining properties of novel social categories (Diesendruck \& Eldror, 2011). A second possible interpretation is that perhaps in other assessments of essentialism, the expected prominence of race over ethnicity would be evident. In particular, recent studies suggest that cultural learning likely influences the relative standing of categories vis-à-vis each other (Diesendruck, Birnbaum, Deeb, \& Segall in press). That is, had we directly asked North American children to determine whether racial or ethnic categories are more objectively correct, perhaps they would have responded differently from the way they responded here, where they were asked about each category in isolation. Finally, it is important to acknowledge that there might be pockets of populations in the New York City area in which children might indeed view the difference between Jews and Arabs in much the same way as Israelis do, that is, as an ethnic divide. These are potential limitations of this study that could be amended in future work. Importantly, these potential methodological shortcomings notwithstanding, this study still revealed meaningful cross-cultural differences in how children in Israel and New York conceptualized racial and ethnic categories. Based on the findings by Rhodes and Gelman (2009) and Diesendruck et al. (in press), it is reasonable to assume that had we sampled children from more "typical" North American communities, or had we used relative measures of essentialism, then the differences between the two countries would be even more substantial.

In conclusion, the findings from this crosscultural developmental study on children's beliefs about the objectivity of various social categories provide novel information on the factors contributing to the essentialization of social categories. In a nutshell, gender was viewed by children from two different countries and ages as objectively correct, 
perhaps attesting to its evolutionary primacy. Occupation, in turn, was viewed more flexibly by children from both countries and across development, intimating that neither its definitional features (i.e., role-based properties) nor cultural factors significantly enhance its essentialist status. Finally, the results suggest that race and ethnicity become targets of essentialism, not strictly due to some a priori intrinsic properties they might contain, but rather due to their particular cultural significance. A further potential contribution of this conclusion is more practical in nature. Namely, given the considerable evidence linking essentialist beliefs to intergroup attitudes in adults, it would be valuable to track this relation from earlier on in development. As a first step, this could be done by examining correlations between children's essentialist beliefs and their implicit or explicit attitudes toward target social categories. If such relations were to be found, then elucidating the role of cultural forces in shaping children's essentialist beliefs about social categories may prove helpful in taming the development of negative and prejudicial intergroup attitudes.

\section{References}

Astuti, R., Solomon, G. E., \& Carey, S. (2004). Constraints on conceptual development. Monographs of the Society for Research in Child Development, 69(3, Serial No. 277).

Atran, S. (1995). Causal constraints on categories and categorical constraints on biological reasoning across cultures. In D. Sperber, D. Premack, \& A. Premack (Eds.), Causal cognition: A multidisciplinary debate (pp. 205-233). Oxford, England: Oxford University Press.

Baron, A. S., \& Banaji, M. R. (2006). The development of implicit attitudes: Evidence of race evaluations from ages 6 and 10 and adulthood. Psychological Science, 17, 53-58. doi:10.1111/j.1467-9280.2005.01664.x

Bar-Tal, D., \& Teichman, Y. (2005). Stereotypes and prejudice in conflict: Representation of Arabs in Israeli Jewish society. Cambridge, England: Cambridge University Press.

Bigler, R. S., \& Liben, L. S. (2007). Developmental intergroup theory: Explaining and reducing children's social stereotyping and prejudice. Current Directions in Psychological Science, 16, 162-166. doi:10.1111/j.1467-8721.2007.00496.x

Birnbaum, D., Deeb, I., Segall, G., Ben-Eliyahu, A., \& Diesendruck, G. (2010). The development of social essentialism: The case of Israeli children's inferences about Jews and Arabs. Child Development, 81, 757-777. doi: 10.1111/j.1467-8624.2010.01432.x

Cosmides, L., Tooby, J., \& Kurzban, R. (2003). Perceptions of race. Trends in Cognitive Sciences, 7, 173-178. doi:10.1016/ S1364-6613(03)00057-3

Deeb, I., Segall, G., Birnbaum, D., Ben-Eliyahu, A., \& Diesendruck, G. (2011). Seeing isn't believing: The effect of intergroup exposure on children's essentialist beliefs about ethnic categories. Journal of Personality and Social Psychology, 101, 1139-1156. doi:10.1037/a0026107

del Rio, M. F., \& Strasser, K. (2011). Chilean children's essentialist reasoning about poverty. British Journal of Developmental Psychology, 29, 722-743. doi:10.1348/ 2044-835X.002005

Diesendruck, G., Birnbaum, D., Deeb, I., \& Segall, G. (in press). Learning what is essential: Relative and absolute changes in children's beliefs about the heritability of ethnicity. Journal of Cognition and Development. doi: 10.1080/15248372.2012.691142

Diesendruck, G., \& Eldror, E. (2011). What children infer from social categories. Cognitive Development, 26, 118126. doi:10.1016/j.cogdev.2010.11.001

Diesendruck, G., \& Haber, L. (2009). God's categories: The effect of religiosity on children's teleological and essentialist beliefs about categories. Cognition, 110, 100 114. doi:10.1016/j.cognition.2008.11.001

Diesendruck, G., \& haLevi, H. (2006). The role of language, appearance, and culture in children's social category based induction. Child Development, 77, 539-553. doi:10.1111/j.1467-8624.2006.00889.x

Dunham, Y., Baron, A. S., \& Carey, S. (2011). Consequences of "minimal" group affiliations in children. Child Development, 82, 793-811. doi:10.1111/j.1467-8624.2011.01577.x

Fodor, J. A. (1998). Concepts: Where cognitive science went wrong. New York: Oxford University Press.

Gelman, S. A. (2003). The essential child: Origins of essentialism in everyday thought. New York: Oxford University Press.

Gelman, S. A., Heyman, G. D., \& Legare, C. H. (2007). Developmental changes in the coherence of essentialist beliefs about psychological characteristics. Child Development, 78, 757-774. doi:10.1111/j.1467-8624.2007.01031.x

Gelman, S. A., Taylor, M. G., \& Nguyen, S. P. (2004). Mother-child conversations about gender: Understanding the acquisition of essentialist beliefs. Monographs of the Society for Research in Child Development, 69(1, Serial No. 275).

Gil-White, F. J. (2001). Are ethnic groups biological "species" to the human brain? Essentialism in our cognition of some social categories. Current Anthropology, 42, 515554. doi:10.1086/321802

Haslam, N., Rothschild, L., \& Ernst, D. (2002). Are essentialist beliefs associated with prejudice? British Journal of Social Psychology, 41, 87-100. doi:10.1348/014466602165072

Hirschfeld, L. A. (1996). Race in the making. Cambridge, MA: MIT Press.

Kalish, C. W., \& Lawson, C. A. (2008). Development of social category representations: Early appreciation of roles and deontic relations. Child Development, 79, 577593. doi:10.1111/j.1467-8624.2008.01144.x

Keller, J. (2005). In genes we trust: The biological component of psychological essentialism and its relationship to mechanisms of motivated social cognition. Journal of Personality and Social Psychology, 88, 686-702. doi: 10.1037/0022-3514.88.4.686 
Kinzler, K. D., \& Dautel, J. (2012). Children's essentialist reasoning about language and race. Developmental Science, 15, 131-138. doi:10.1111/j.1467-7687.2011.01101.x

Kinzler, K. D., Shutts, K., \& Correll, J. (2010). Priorities in social categories. European Journal of Social Psychology, 40, 581-592. doi:10.1002/ejsp.739

Morton, T. A., Postmes, T., Haslam, S. A., \& Hornsey, M. J. (2009). Theorizing gender in the face of social change: Is there anything essential about essentialism? Journal of Personality and Social Psychology, 96, 653-664. doi: $10.1037 /$ a0012966

Prentice, D. A., \& Miller, D. T. (2007). Psychological essentialism of human categories. Current Directions in Psychological Science, 16, 202-206. doi:10.1111/j.14678721.2007.00504.x

Rhodes, M., \& Brickman, D. (2011). The influence of competition on children's social categories. Journal of Cognition and Development, 12, 194-221. doi:10.1080/ 15248372.2010 .535230

Rhodes, M., \& Gelman, S. A. (2009). A developmental examination of the conceptual structure of animal, artifact, and human social categories across two cultural contexts. Cognitive Psychology, 59, 244-274. doi:10.1016/ j.cogpsych.2009.05.001
Rhodes, M., Leslie, S., \& Tworek, C. M. (2012). Cultural transmission of social essentialism. Proceedings of the National Academy of Sciences, 109, 13526-13531. doi:10. 1073/pnas.1208951109

Rutland, A., Killen, M., \& Abrams, D. (2010). A new social-cognitive developmental perspective on prejudice: The interplay between morality and group identity. Perspectives on Psychological Science, 5, 279-291. doi:10.1177/1745691610369468

Segall, G., \& Diesendruck, G. (2011, April). The language of essentialism. Poster presented at the biennial meeting of the Society for Research in Child Development, Montreal, Canada.

Taylor, M. G., Rhodes, M., \& Gelman, S. A. (2009). Boys will be boys; cows will be cows: Children's essentialist reasoning about gender categories and animal species. Child Development, 80, 461-481. doi:10.1111/j.1467-8624. 2009.01272.x

Williams, M. J., \& Eberhardt, J. L. (2008). Biological conceptions of race and the motivation to cross racial boundaries. Journal of Personality and Social Psychology, 94, 1033-1047. doi:10.1037/0022-3514.94.6.1033 